\title{
Resilencia y Ansiedad Precompetitiva en nadadores en edad escolar. Un estudio descriptivo.
} Resilience and Precompetitive Anxiety in swimmers of
school age. A descriptive study.

Victor Manuel Arnau Universitat de València

Irene Checa Esquiva Universitat de València

M. Rocio Bohórquez Gómez-Millán Universidad de Sevilla

\section{resumen/alsstract:}

La ansiedad y la resiliencia en deporte han sido objeto de estudio en los últimos años, aunque rara vez se han abordado con practicantes infantiles. El objetivo de este trabajo fue explorar la influencia que el género, la edad, los años de práctica y la resiliencia tienen sobre la ansiedad competitiva en una muestra de nadadores escolares ( $\mathrm{N}=74)$. La resiliencia fue medida con una adaptación española del CD-RISC, mientras que para medir la ansiedad competitiva se utilizó la versión española del CSAI-2R. También se exploraron los datos sociodemográficos (edad, sexo, años de experiencia y nivel competitivo). Para el análisis de datos se realizaron pruebas no paramétricas (Kruskal-Wallis y U de Mann Whitney), además de correlación de Pearson. Los resultados mostraron que el género, la edad de los nadadores y los años practicando natación no guardan relación con los niveles de ansiedad competitiva de los nadadores en edad escolar evaluados, mientras que el grado de resiliencia mostró una relación positiva con los niveles de autoconfianza, una relación negativa con los niveles de ansiedad cognitiva, y ninguna relación con la ansiedad somática. Estos resultados demuestran que el entrenamiento en la resiliencia de los jóvenes nadadores puede reducir sus niveles de ansiedad y mejorar su experiencia deportiva.

Anxiety and resilience in sports have been the subject of study in recent years, although they have rarely been addressed in child stages. The objective of this work was to explore the influence that gender, age, swimming years and resilience have on competitive anxiety in a sample of school swimmers $(N=74)$. Resilience was measured with a Spanish adaptation of the CD-RISC, while the Spanish version of the CSAI-2R was used to measure competitive anxiety. Sociodemographic data (age, sex, swimming years and competitive level) were also explored. For the analysis of data, non-parametric tests were performed (Kruskal-Wallis and Mann Whitney U), in addition to Pearson's correlation. The results showed that the gender, the age of the swimmers and the years swimming were not related to the levels of competitive anxiety of the swimmers of school age evaluated, while the degree of resilience showed a positive relationship with the levels of self-confidence, a negative relationship with levels of cognitive anxiety, and no relationship with somatic anxiety. These results show that resilience training of young swimmers can reduce their levels of anxiety and improve their sporting experience.

\section{palabras clave/keywords:}

Resiliencia, ansiedad, nadadores, autoconfianza.

Resiliencia y Ansiedad Precompetitiva en nadadores en edad escolar. Un estudio descriptivo. 


\section{Introducción}

La resiliencia es un constructo psicológico que en los últimos años ha recibido una importante atención por parte de la investigación psicológica en diferentes ámbitos como el de la salud (Rosenberg et al, 2015; Souri y Hasanirad, 2011), el de la educación (Condly, 2006; Jennings, Snowberg, Coccia y Greenberg, 2011) o el laboral (Abbott, Klein, Hamilton y Rosenthal, 2009). Este concepto, que nació en física hace referencia a la elasticidad de los materiales; es posteriormente cuando el término resiliencia se utiliza para designar a la capacidad humana que permite no solamente sobrevivir a situaciones adversas, dolorosas o difíciles, sino además enriquecerse con ellas (Cyrulnik et al., 2004).

Existen numerosas definiciones de resiliencia y no parece haber un consenso en torno a este término, lo que parece indicar que es un fenómeno complejo, multicausal y multifactorial en el que convergen las distintas dimensiones del ser humano (García-Vesga y Domínguezde la Ossa, 2013). Sí parece haber acuerdo en que la resiliencia es un proceso y no un estado (Martínez y Vásquez-Bronfman, 2006) y que puede ser enseñada y adquirida a través de un ambiente esperanzador y optimista (Campo, Granados, Muñoz, Trujillo, y Rodríguez, 2012). Algunos autores han planteado que la resiliencia está constituida por dos dimensiones: (a) resistencia a la destrucción y protección frente a presiones, y (b) capacidad de construir y crear una vida digna a pesar de las adversidades (Vanistendael, 1994).

La personalidad resiliente está relacionada con el concepto de personalidad resistente o "hardiness", el cual hace referencia a la personalidad fuerte y dureza mental de algunos deportistas (Jaenes, Godoy, y Román, 2009). La personalidad resistente es un constructo multidimensional con tres componentes: compromiso, control y desafío o estímulo ante las adversidades (Kobasa, 1979, 1982). Una puntuación alta en compromiso se relaciona con encontrar significado a lo que se hace y con la capacidad para manejar sucesos estresantes (Sousa, 2006; Kobasa, 1979). Las personas que puntúan alto en el componente de control tienen una alta percepción de su capacidad de manejar su entorno y las acciones que les suceden. Aquellas personas con puntuaciones altas en desafío se caracterizan por confiar en que el cambio, el crecimiento y el aprendizaje les ayude a mejorar sus vidas y enriquecerse personalmente (Kobasa, 1979; Jaenes et al., 2009).

Algunos estudios plantean que los deportistas con personalidad resistente presentan menores niveles de ansiedad precompetitiva y mayores niveles de autoconfianza (Bonanno, 2004; González, Moore, Newton y Galli, 2016). Esto podría ser debido a que estos deportistas manejan la adversidad mucho mejor que los demás (Hanton, Evans y Neil, 2003) o a que se han visto previamente involucrados en programas de entrenamiento en toma de decisiones y estrategias de afrontamiento (Cowden, Fuller y Anshel, 2014); los resistentes saben que se puede crecer y aprender tanto de experiencias positivas como negativas, de forma que evalúan situaciones potencialmente estresantes como menos amenazadoras, disminuyendo así la ansiedad. Además, presentan menos alteraciones psicosomáticas a nivel físico y psíquico ante una situación estresante (Jaenes et al., 2009). 
La ansiedad competitiva es un concepto multidimensional relacionado inversamente con el rendimiento y conformado por tres factores: ansiedad cognitiva, ansiedad somática y autoconfianza (Martens, Vealey y Burton, 1990). El tipo específico de ansiedad que aparece antes de una competición se denomina ansiedad precompetitiva (Cox, 2009). La ansiedad cognitiva se refiere a los pensamientos negativos que navegan en la mente del deportista, la preocupación sobre su ejecución o rendimiento deportivo, o la desconcentración momentos antes de competir. Así, algunos deportistas padecen miedo, pánico, inquietud, incluso obsesiones. La ansiedad somática se manifiesta a través de ciertos síntomas corporales relacionados con la actividad del sistema nervioso autónomo (taquicardia, sudoración, temblor, etc.) (Rodríguez y Granero-Gallegos, 2004). Por último, la autoconfianza es la creencia de una persona acerca de su capacidad para ejecutar con éxito la conducta requerida para producir un determinado resultado (Garcés de los Fayos y Cantón, 2007); la creencia en uno mismo, la confianza en la propia preparación para superar con éxito los obstáculos enfrentados (Rodríguez y Granero-Gallegos, 2004). La autoconfianza en los momentos previos y durante la competición se ha relacionado con bajos niveles de ansiedad y un mejor nivel de ejecución (Craft, Magyar, Becker, y Feltz, 2003).

Entre las disciplinas deportivas en las que puede aparecer la ansiedad competitiva se encuentra la natación, y así lo han corroborado los estudios en dicho campo (Canton, Checa y Ortín, 2010; Grossbard, Smith, Smoll y Cumming, 2009). En este sentido, ya hay ciertas técnicas, como el desarrollo de la autoeficacia (Márquez, 2006), o el establecimiento de objetivos (Cantón et al., 2010), que han demostrado su utilidad en el control de la ansiedad competitiva.

Los estudios que han analizado la ansiedad competitiva en jóvenes nadadores en función del género, muestran en unos casos que los hombres presentan menores niveles de ansiedad cognitiva y somática que las mujeres, así como una mayor autoconfianza (Grau, 2014). Además, otros autores señalan que esta mayor ansiedad de las mujeres podría explicarse porque tienen un mayor miedo a fallar y a ser socialmente evaluadas (Grossbard et al., 2009) y que además perciben la ansiedad como debilitadora de su rendimiento (Thatcher, Thatcher y Dorling, 2004). Sin embargo, un estudio más reciente contradice los resultados anteriores, encontrando que los hombres perciben un mayor nivel de ansiedad, pero lo interpretan de forma más facilitadora (Massengale, 2012). Finalmente, otros estudios no encuentran relación alguna entre el género y la ansiedad competitiva (Pozo, 2007).

En cuanto al nivel competitivo, los nadadores de más alto nivel, han mostrado una mayor autoconfianza (Jones, 1995) y una interpretación más facilitadora de los tres factores de ansiedad (Pozo, 2007).

Sin embargo, no se ha encontrado ningún estudio que analice la relación entre los factores de la ansiedad precompetitiva y la resiliencia en una muestra específica de nadadores en edad escolar. Por ese motivo, el objetivo de este trabajo es indagar en la influencia que el género, la edad, los años de práctica y la resiliencia tienen sobre la ansiedad competitiva de una muestra de nadadores en edad escolar. 


\section{Método}

\section{Participantes}

La muestra estuvo compuesta por 74 sujetos, de los cuales 29 fueron mujeres conformando el $39.19 \%$ del total y 45 hombres que suponen el $60.81 \%$. Todos los deportistas practican natación y la edad de los participantes se sitúa entre los 8 y los 14 años $(M=11,22 ; S D=1,67)$ y llevan entre 1 y 12 años de experiencia $(M=4,89 ; \mathrm{SD}=2,77)$. Respecto al nivel competitivo, 36 nadadores compiten en nivel autonómico (48.6\%), 22 a nivel provincial (29.7\%) y 16 nadadores compiten a nivel nacional $(21.6 \%)$.

\section{Instrumentos}

La batería de cuestionarios a cumplimentar reunía evaluación de la resiliencia, ansiedad precompetitiva y un cuestionario de datos sociodemográficos que incluía edad, sexo, años de experiencia nadando y nivel competitivo.

La resiliencia fue evaluada con la versión breve de la CD-RISC en su adaptación española (Notario et al., 2011); en esta adaptación se parte de los 35 ítems que conforman la escala original (Connor y Davidson, 2003) y se reducen a 10 ítems agrupados en una única dimensión. Se trata de un instrumento de autoinforme con escala de respuesta aditiva tipo Likert de cinco puntos que oscila entre 0 (nunca) y 4 (casi siempre), de forma que a mayor puntuación, mayores son los niveles de resiliencia. El instrumento ha mostrado en otros estudios niveles adecuados de fiabilidad $(\alpha=0,85)$ y validez cuando se ha utilizado en muestra española universitaria (Notario et al., 2011). La fiabilidad del instrumento en la muestra actual resulta muy alta $(\alpha=0,969)$.

Para la medición de la ansiedad competitiva se utilizó la versión española del CSAI-2R (Andrade, Lois y Arce, 2007). Está compuesto por 17 ítems que se desglosan en tres subescalas: ansiedad cognitiva, ansiedad somática y autoconfianza. La forma de respuesta es tipo Likert de cuatro puntos, siendo el 1 el grado de mayor desacuerdo y el 4 el de mayor acuerdo con cada afirmación. Con el fin de evitar cierta ambigüedad se combinaron las traducciones directas e inversas de los ítems (Andrade, Arce y Seoane, 2002; Arce, Andrade, y Seoane, 2000). La fiabilidad de este instrumento en la muestra actual resulta satisfactoria $(\alpha=0,812)$.

\section{Procedimiento}

La recogida de datos se llevó a cabo en dos días. En primer lugar se solicitó a los tutores legales de los deportistas, a través de sus clubes, el consentimiento informado para la participación en la investigación; en base a aquellos que consintieron participar, se contactó con los deportistas a través de sus entrenadores. Se informó además a los deportistas que al participar lo hacían de forma voluntaria y anónima, se les insistió en que no había respuestas correctas o incorrectas y que debían responder de la forma más sincera posible. Para agilizar el proceso, con cada grupo se fueron leyendo y explicando uno por uno los enunciados a la vez que lo respondían. No obstante, si algún deportista se retrasaba se volvía a propiciar una explicación. 


\section{Análisis de Datos}

De forma previa al análisis de datos, se calcularon los factores del CSAI-2R y se dividió la muestra en 4 grupos en función de 4 niveles de resiliencia. Los grupos de resiliencia se conformaron en función de la distribución de sujetos de la muestra: el grupo de resiliencia muy baja los formaron deportistas con percentiles entre el 0 y el 25 (puntuaciones directas 0 a 18.75); el grupo de resiliencia baja por nadadores entre el percentil 25 y el 50 (puntuaciones directas entre 18.76 y 34.50 ); el grupo de resiliencia alta se compuso de atletas con percentiles entre el 50 y el 75 (puntuaciones directas de 34.50 a 44); y el último grupo de resiliencia muy alta lo formaron deportistas con percentiles entre el 75 y el 100 (puntuaciones directas de 44.01 en adelante).

Tabla 1. Ansiedad y Resiliencia en función del Género

\begin{tabular}{|c|c|c|c|c|c|c|}
\hline & Género & $\begin{array}{l}\text { Rango } \\
\text { promedio }\end{array}$ & $\begin{array}{c}\text { Suma de } \\
\text { rangos }\end{array}$ & $\begin{array}{l}\text { UMann- } \\
\text { Whitney }\end{array}$ & Z & $\begin{array}{c}\text { Sig. } \\
\text { asintótica } \\
\text { (bilateral) }\end{array}$ \\
\hline \multirow{3}{*}{ Ans_C } & Hombre & 38.07 & 1713.00 & \multirow{3}{*}{627} & \multirow{3}{*}{-.284} & \multirow{3}{*}{.776} \\
\hline & & & & & & \\
\hline & Mujer & 36.62 & 1062.00 & & & \\
\hline \multirow{3}{*}{ Ans_S } & Hombre & 36.62 & 1648.00 & \multirow{3}{*}{613} & \multirow{3}{*}{-.439} & \multirow{3}{*}{.661} \\
\hline & & & & & & \\
\hline & Mujer & 38.86 & 1127.00 & & & \\
\hline \multirow{3}{*}{ Autoc } & Hombre & 36.28 & 1632.50 & \multirow{3}{*}{597.5} & \multirow{3}{*}{-.613} & \multirow{3}{*}{.540} \\
\hline & & & & & & \\
\hline & Mujer & 39.40 & 1142.50 & & & \\
\hline \multirow{3}{*}{ Resiliencia } & Hombre & 37.16 & 1672.00 & \multirow{3}{*}{637} & \multirow{3}{*}{-.172} & \multirow{3}{*}{.864} \\
\hline & & & & & & \\
\hline & Mujer & 38.03 & 1103.00 & & & \\
\hline
\end{tabular}

Nota: Ans_C; ansiedad Cognitiva; Ans_S: Ansiedad Somática; Autoc: Autonconfianza; 
Tabla 2. Correlaciones entre la edad, los años de práctica, la ansiedad y la resiliencia

\begin{tabular}{lccccc}
\hline \multirow{2}{*}{ Edad } & & Ans_C & Ans_S & Autoc & Resiliencia \\
\cline { 2 - 6 } & $\mathrm{r}$ & -.128 & -.063 & .022 & -.094 \\
\cline { 2 - 6 } Años de práctica & $\mathrm{p}$ & .278 & .593 & .856 & .424 \\
\cline { 2 - 6 } & $\mathrm{r}$ & .018 & .137 & -.033 & -.069 \\
\hline & $\mathrm{p}$ & .880 & .245 & .781 & .559 \\
\hline
\end{tabular}

Nota: Ans_C; ansiedad Cognitiva; Ans_S: Ansiedad Somática; Autoc: Autonconfianza; r: correlación de Pearson; p: significación bilateral.

A continuación se realizaron pruebas de normalidad y homoscedasticidad de la muestra con el fin de tomar decisiones acerca de las pruebas estadísticas a utilizar. Se determinó una distribución no normal de la muestra, así como la no homogeneidad de varianzas para las variables principales del estudio (pruebas de Kolmogorov-Smirnov y Levene, $p<.05$ ). Por ello se decidió utilizar pruebas no paramétricas en los análisis estadísticos realizados para este estudio.

Para la definición de la línea base del grupo evaluado, se llevó a cabo un análisis de frecuencias. Para analizar la influencia del género en la ansiedad cognitiva, ansiedad somática, autoconfianza y resiliencia se realizaron pruebas no paramétricas de comparación de dos grupos independientes U de Mann-Whitney. Con el propósito de averiguar la influencia de la edad y el número de años compitiendo en la ansiedad cognitiva, ansiedad somática, autoconfianza y resiliencia, se realizó una correlación de Pearson, ya que es un análisis para la muestra total y su $\mathrm{N}$ permite realizar prueba paramétrica. Por último, para analizar la influencia de los niveles de resiliencia sobre la ansiedad cognitiva, ansiedad somática y autoconfianza, se emplearon pruebas no paramétricas de comparación de más de dos grupos independientes Kruskal-Wallis y prueba U de Mann-Whitney para las comparaciones a posteri. Todos los datos se analizaron con software estadístico SPSS 22.0 (IBM, 2013).

Tabla 3. Resultados para ansiedad cognitiva. somática y autoconfianza en función de los niveles de resiliencia

$$
\text { Ansiedad cognitiva Ansiedad somática Autoconfianza }
$$

\begin{tabular}{lccccccccc} 
& $\mathrm{X}^{2}$ & $\mathrm{Gl}$ & $\mathrm{p}$ & $\mathrm{X}^{2}$ & $\mathrm{gl}$ & $\mathrm{p}$ & $\mathrm{X}^{2}$ & $\mathrm{Gl}$ & $\mathrm{p}$ \\
\hline $\begin{array}{l}\text { Resiliencia } \\
\text { (niveles) }\end{array}$ & 7.693 & 3 & $.05^{*}$ & 4.08 & 3 & .25 & 34.14 & 3 & $.00^{* *}$ \\
\hline
\end{tabular}

Nota: ${ }^{*} \mathrm{p}<.05 . * * \mathrm{p}<.01$ 
Tabla 4. Estadísticos descriptivos e inferenciales de los factores de ansiedad en función de los niveles de resiliencia

\begin{tabular}{|c|c|c|c|c|}
\hline Resiliencia & & Ansiedad Cognitiva & Ansiedad Somática & Autoconfianza \\
\hline \multirow{2}{*}{ Muy Baja } & $\mathrm{M}$ & 14.11 & 19.17 & 10.44 \\
\hline & DT & 3.85 & 3.88 & 4.05 \\
\hline \multirow{2}{*}{ Baja } & $\mathrm{M}$ & 11.89 & 17.74 & 14.68 \\
\hline & DT & 2.73 & 4.24 & 3.56 \\
\hline \multirow{2}{*}{ Alta } & $\mathrm{M}$ & 12.35 & 18.06 & 17.06 \\
\hline & DT & 2.50 & 5.04 & 2.66 \\
\hline \multirow{2}{*}{ Muy Alta } & $\mathrm{M}$ & 11.20 & 15.80 & 18.65 \\
\hline & DT & 3.27 & 4.94 & 1.46 \\
\hline \multirow{2}{*}{ MB-B } & $\mathrm{U}$ & 100.50 & 131.50 & 69.00 \\
\hline & $\mathrm{p}$ & $.031^{*}$ & .277 & $.000^{* *}$ \\
\hline \multirow{2}{*}{ MB-A } & $\mathrm{U}$ & 101.50 & 130.00 & 31.00 \\
\hline & $\mathrm{p}$ & .08 & .44 & $.000^{* *}$ \\
\hline \multirow{2}{*}{ MB-MA } & $\mathrm{U}$ & 96.00 & 107.00 & 20.00 \\
\hline & $\mathrm{p}$ & $.01^{*}$ & $.03^{*}$ & $.000^{* *}$ \\
\hline \multirow{2}{*}{ B-A } & $\mathrm{U}$ & 147.50 & 158.00 & 94.00 \\
\hline & $\mathrm{p}$ & .654 & .91 & $.03^{*}$ \\
\hline \multirow{2}{*}{ B-MA } & $\mathrm{U}$ & 170.50 & 162.50 & 65.00 \\
\hline & $\mathrm{p}$ & .58 & .43 & $.000^{* *}$ \\
\hline \multirow{2}{*}{ A-MA } & $\mathrm{U}$ & 137.50 & 141.00 & 108.50 \\
\hline & $\mathrm{p}$ & .31 & .37 & $.05^{*}$ \\
\hline
\end{tabular}

MB: Resiliencia muy baja; B: Resiliencia Baja; A: Resiliencia Alta; MA: Resiliencia muy Alta; M= Media; DT: Desviación Típica; U: U de Mann-Whitney; p: significación; *:p<.05; **:p<.01

\section{Resultados}

Respecto a la distribución de los resultados obtenidos con el cuestionario CD-RISC, que mide resiliencia, la muestra obtiene los siguientes resultados: 18 nadadores $(24.3 \%)$ obtienen una puntuación muy baja en la variable resiliencia; 19 nadadores $(25.7 \%)$ obtienen una puntuación baja; 17 nadadores $(23 \%)$ obtienen una puntuación alta en resiliencia; mientras que 20 nadadores (27\%) obtienen una puntuación muy alta en la variable resiliencia. 
En relación al género y para comprobar la influencia de esta variable en la ansiedad cognitiva, ansiedad somática, autoconfianza y resiliencia se realizaron pruebas no paramétricas de comparación de grupos independientes $U$ de Mann-Whitney. Los resultados, que se muestran en la Tabla 1 demuestran que no hay diferencias significativas en ninguna de las variables analizadas en función del género: ni en los niveles de ansiedad cognitiva $(\mathrm{Z}=-.284, p$ $>.05)$, ni en ansiedad somática $(Z=-.439, p>.05)$, ni en autoconfianza $(Z=-.613, p>.05)$, ni en resiliencia $(Z=-.172, p>.05)$ en función del género.

En lo relativo a la edad y el número de años compitiendo, los resultados indicaron que no son variables que estén relacionadas en esta muestra con la ansiedad cognitiva, ansiedad somática, autoconfianza o la resiliencia (ver Tabla 2).

Tras comprobar que ni el género ni los años de práctica ni la edad de los nadadores estaban relacionadas con la resiliencia ni con la ansiedad precompetitiva, se analizó la posible influencia que los niveles de resiliencia pudieran tener en los factores de ansiedad precompetitiva. Como se puede observar en la Tabla 3 existen diferencias significativas en los niveles de ansiedad cognitiva $\left(\chi_{(3,74)}=7.69 . p<.05\right)$ y en la autoconfianza de los nadadores $\left(\chi 2_{(3.74)}\right.$ $=34.14, p<.01)$ en función de su nivel de resiliencia. Sin embargo, no existen diferencias significativas en los niveles de ansiedad somática de los nadadores en función de su nivel de resiliencia $\left(\chi_{(3,74)}=4.08, p=.25\right)$.

Para aquellos factores que han mostrado diferencias significativas en función de los niveles de resiliencia se han llevado a cabo análisis a posteriori dos a dos con los siguientes resultados (Tabla 4). Respecto a la ansiedad cognitiva, los nadadores con niveles de resiliencia muy bajos presentan puntuaciones mayores que aquellos con niveles bajo ( $\mathrm{U}=100.50$, $p<.031)$ o muy alto de resiliencia $(\mathrm{U}=96.00, p<.01)$. En relación a la ansiedad somática los nadadores con niveles de resiliencia muy bajos la sufren significativamente más que aquellos con niveles de resiliencia muy altos $(\mathrm{U}=107.00, \mathrm{p}<.03)$. Finalmente, respecto a la autoconfianza es donde más diferencias se encuentran entre grupos. Los nadadores con niveles de resiliencia muy bajos presentan una menor autoconfianza que aquellos con niveles bajos $(\mathrm{U}=69.00, p<.01)$, niveles altos $(\mathrm{U}=31.00, p<.01)$ y niveles muy altos $(\mathrm{U}=20.00, p<.01)$. Además, los nadadores con niveles de resiliencia bajos presentan una menor autoconfianza que los grupos con resiliencia alta $(\mathrm{U}=94.00, p<.03)$ y muy alta $(\mathrm{U}=65.00, p<.01)$. Por último los nadadores con niveles de resiliencia altos presentan una mayor autoconfianza que aquellos con niveles de resiliencia muy altos $(\mathrm{U}=108.50, p<.05)$

\section{Discusión}

El objetivo de este trabajo era indagar en la influencia que el género, la edad, los años de experiencia en la natación y la resiliencia pudieran tener sobre la ansiedad competitiva de una muestra de nadadores en edad escolar.

Los resultados mostraron que el género, la edad de los nadadores y los años de práctica no guardan relación con los niveles de ansiedad competitiva de los nadadores en edad escolar evaluados. Para el género, estudios previos mostraron resultados contradictorios al señalar unos (Grau, 2004; Grossbard et al., 2009; Thatcher, Thatcher y Dorling, 2004) mayor an- 
siedad cognitiva y somática en mujeres deportistas así como menor autoconfianza que los varones, y otros (Massengale, 2012) mayor ansiedad -pero facilitadora- en hombres. Pozo (2007) no encontró relación entre ambas variables, resultados coherentes con los hallados en este estudio. Morillo, Reigal y Hernández-Mendo (2016) explican las diferencias de género en ansiedad competitiva en función de otras variables como el nivel motivacional o las estrategias de afrontamiento empleadas; estas últimas, dependientes de procesos de aprendizaje que quizás sean disímiles entre géneros dados los estereotipos imperantes (Tabernero y Márquez, 1993) generando las diferencias aportadas en algunos estudios.

En lo relativo a la edad, Tabernero y Márquez (1993) encontraron diferencias en las causas de la ansiedad competitiva en función de ésta, variando desde motivos propios de la interacción a motivos más relacionados con la competición y su gestión psicológica a medida que los deportistas crecían. Por su parte, Bohórquez y Checa (2017), encontraron que los deportistas -jugadores de fútbol y tenis- más jóvenes puntuaron más en la escala preocupación del SAS-2 (Ramis, Torregrosa, Viladrich y Cruz, 2010) que sus compañeros mayores. Es posible que las diferencias inherentes al diseño de los cuestionarios empleados generen las discrepancias halladas en los resultados de los diferentes estudios, ya que el CSAI-2 se diseñó para población general mientras que el SAS-2 es específico para deporte de iniciación, resultando posiblemente diferentes las fuentes de ansiedad en función de la edad de los atletas; aunque éste es un aspecto que habrá que abordar en futuros estudios.

La experiencia compitiendo no resultó tener influencia sobre la ansiedad competitiva de esta muestra de jóvenes nadadores. Parece que es el nivel competitivo adquirido, y no la edad, la que influye en la intensidad de la ansiedad (Jones, 1995) y la interpretación de la misma por parte de los deportistas (Pozo, 2007).

Estas variables sociodemográficas tampoco tuvieron impacto sobre la resiliencia. Estos resultados son coherentes con los hallados para población general en lo relativo al género (Prado y del Águila, 2003; Sun y Stewart, 2007) y la edad (Sun y Stewart, 2007). No se han encontrado estudios que relacionen género, edad o años de experiencia deportiva y resiliencia en población deportista, por lo que no se pueden extraer más conclusiones acerca de la relación entre ambas en este contexto.

En cuanto a la resiliencia, se quiso saber si ésta influiría en la ansiedad competitiva de los jóvenes nadadores. Los resultados mostraron que aquellos nadadores con niveles muy bajos de resiliencia mostraron mayor ansiedad cognitiva que sus compañeros con mayores niveles de resiliencia, resultado coherente con los encontrados previamente por Cowden, et al. (2014), González, et al. (2016) o Hanton, et al. (2003) en deportistas adultos. En la misma línea, los diferentes niveles de resiliencia generaron diferentes puntuaciones en ansiedad, de tal modo que, a menores niveles de resiliencia, menor autoconfianza mostraban los nadadores, lo que podría atribuirse según diferentes autores (Bonanno, 2004; Hanton, et al. ,2003) a una interpretación facilitadora de la ansiedad competitiva en deportistas resilientes. Por otro lado, la resiliencia no influye en la ansiedad somática mostrada por los nadadores de esta muestra, del mismo modo que ya ocurriera en estudios previos con deportistas adultos (Hanton et al, 2003). 
Entre las principales limitaciones de este estudio cabe destacar el tamaño de la muestra que limita el impacto de las conclusiones obtenidas, así como el empleo de cuestionarios de evaluación no específicos para población infantil. En el futuro, sería interesante conocer la relación entre la resiliencia y las estrategias de afrontamiento utilizadas por los deportistas, debido a la comprobada relación entre éstas y la experiencia de ansiedad (Cantón, Checa y Vellisca, 2015; Ramis, Torregrosa y Cruz, 2013).

Entre las aportaciones de este estudio destaca la relación entre una baja resiliencia y su correlato en mayor ansiedad competitiva y menor autoconfianza por parte de los jóvenes nadadores. Esta relación, sumada a las consecuencias ya conocidas de la ansiedad competitiva en las etapas de iniciación invita al diseño e implementación de programas de intervención que promocionen el desarrollo de la resiliencia en los jóvenes deportistas.

\section{Referencias}

Abbott, J. A., Klein, B., Hamilton, C., y Rosenthal, A. (2009). The Impact of Online Resilience Training for Sales Managers on Wellbeing and Work Performance. E-Journal of Applied Psychology. 5(1), 89-95.

Andrade, E., Arce, C., y Seoane, G. (2002). Adaptación al español del cuestionario «Perfil de los estados de ánimo» en una muestra de deportistas. Psicothema. 14, 708-713.

Andrade, E. M., Lois, G., y Arce, C. (2007). Propiedades psicométricas de la versión española del Inventario de Ansiedad Competitiva CSAI-2R en deportistas. Psicothema, 1911), 150-155.

Arce, C., Andrade, E., y Seoane, G. (2000). Problemas semánticos en la adaptación del POMS al castellano. Psicothema, $12,47-51$.

Bohórquez, M., y Checa, I. (2017). Factores psicosociales relacionados con la ansiedad competitiva de los deportistas en etapas de formación. Cultura, Ciencia y Deporte, 12(36), 205-210.

Bonanno, G. A. (2004). Loss, trauma, and human resilience: have we underestimated the human capacity to thrive after extremely aversive events? American psychologist, 5911), 20. http://dx.doi.org/10.1037/0003-066X.59.1.20

Campo, R., Granados, L. F., Muñoz, L., Rodríguez, M. S., y Trujillo, S. (2012). Characterization of the research, the intervention and the theoretical advances in resiliency in the universities in Colombia. Universitas Psychologica, $11(2), 545-557$.

Cantón, E., Checa, I., y Ortín, F. J. (2010). Intervención psicológica con un nadador de medio acuático abierto. Cuadernos de psicología del deporte, 9(2), 57-62.

Cantón, E., Checa, I. y Vellisca, M. Y. (2015). Bienestar psicológico y ansiedad competitiva: el papel de las estrategias de afrontamiento. Revista Costarricense de Psicología, 34(2), 71-78.

Condly, S. J. (2006). Resilience in children: A review of literature with implications for education. Urban education. 41(3). 211-236. https://doi.org/10.1177/0042085906287902

Connor, K., y Davidson, J. (2003). Development of a new resilience scale: the Connor-Davidson Resilience Scale (CDRISC). Depress Anxiety, 18(2), 76-82. https://doi.org/10.1002/da.10113

Cowden, R. G., Fuller, D. K., y Anshel, M. H. (2014). Psychological Predictors of Mental Toughness in Elite Tennis: An Exploratory Study in Learned Resourcefulness and Competitive Trait Anxiety. Perceptual and Motor Skills, 1193), 661-678. https://doi.org/10.2466/30.PMS.119c27z0 
Cox, R. H. (2009). Psicología del deporte: Conceptos y sus aplicaciones. Madrid: Médica Panamericana.

Craft, L. L., Magyar, T. M., Becker, B. J., y Feltz, D. L. (2003). The relationship between the Competitive State Anxiety Inventory-2 and sport performance: A meta-analysis. Journal of sport and exercise psychology. 25(1). 44-65. https://doi.org/10.1123/jsep.25.1.44

Cyrulnik, B (2004). Resiliencia, la fuerza de la vida. Guatemala: Editorial Egap

Garcés de Los Fayos, E. J., y Cantón, E. (2007). Un modelo teórico-descriptivo del burnout en deportistas: Una propuesta tentativa. Informació Psicòlogica, 91-92, 12-22.

García-Vesga, M. C., y Domínguez-de la Ossa, E. (2013). Desarrollo teórico de la Resiliencia y su aplicación en situaciones adversas: Una revisión analítica. Revista Latinoamericana de Ciencias Sociales. Niñez y Juventud, 11(1), 63-77.

González, S. P., Moore, E. W. G., Newton, M., y Galli, N. A. (2016). Validity and reliability of the Connor-Davidson Resilience Scale (CD-RISC) in competitive sport. Psychology of Sport and Exercise, 23, 31-39. https://doi. org/10.1016/j.psychsport.2015.10.005

Grau, J. A. (2014). The effect of breathing techniques and mental imagery training on pre competitive anxiety and selfconfidence of collegiate swimmers. IL: Western Illinois University.

Grossbard, J. R., Smith, R. E., Smoll, F. L., y Cumming, S. P. (2009). Competitive anxiety in young athletes: Differentiating somatic anxiety, worry and concentration disruption. Anxiety, Stress and Coping, 22(2), 153-166. https://doi. org/10.1080/10615800802020643

Hanton, S., Evans, L., y Neil, R. (2003) Hardiness and the competitive trait anxiety response. Anxiety, Stress and Coping, 16(2), 167-184. https://doi.org/10.1080/10615806.2003.10382971

IBM Corp. Released 2013. IBM SPSS Statistics for Windows. Version 22.0. Armonk. NY: IBM Corp.

Jaenes, J. C., Godoy. D., y Román, F. M. (2009). Personalidad resistente en maratonianos: un estudio sobre el control. compromiso y desafío de corredoras y corredores de maratón. Revista de Psicología del Deporte, 18, 217-234.

Jones, G. (1995). More than just a game: Research developments and issues in competitive anxiety in sport. British journal of psychology, 86(4), 449-478. http://dx.doi.org/10.1111/j.2044-8295.1995.tb02565.x

Kobasa, S. C. (1979). Stressful life events. Personality and health: an inquiry into hardiness. Journal of Personality and Social Psychology, 37, 1-10. http://dx.doi.org/10.1037/0022-3514.37.1.1

Kobasa, S. C. (1982). Commitment and coping in stress resistance among lawyers. Journal of Personality and Social Psychology, 4, 707-717. http://dx.doi.org/10.1037/0022-3514.42.4.707

Márquez, S. (2006). Estrategias de afrontamiento del estrés en el ámbito deportivo: fundamentos teóricos e instrumentos de evaluación. International Journal of Clinical and Health Psychology, 6(2), 359-378.

Martínez, I., y Vásquez-Bronfman, A. (2006). La resiliencia invisible: infancia. inclusión social y tutores de vida. Barcelona: Gedisia.

Martens, R., Vealey, R. S., y Burton, D. (1990). Competitive Anxiety in Sport. Champaign. IL: Human Kinetics.

Massengale, B. D. (2012). A temporal examination of achievement goals, motivational climate and anxiety in collegiate swimmers. Southern Illinois University at Carbondale.

Morillo, J. P., Reigal, R. E., y Hernández-Mendo, A. (2016). Relaciones entre el perfil psicológico deportivo y la ansiedad competitiva en jugadores de balonmano playa. Revista de Psicología del Deporte. 25(1), 125-128.

Notario-Pacheco, B., Solera-Martínez, M., Serrano-Parra, M. D., Bartolomé-Gutiérrez, R., García-Campayo, J., y Martínez-Vizcaíno, V. (2011). Reliability and validity of the Spanish version of the 10-item Connor-Davidson Resilience Scale (10-item CD-RISC) in young adults. Health and Quality of Life Outcomes, 911), 63. https://doi. org/10.1186/1477-7525-9-63 
Pozo, A. (2007). Intensidad y dirección de la ansiedad competitiva y expectativas de resultados en atletas y nadadores. Revista de psicología del deporte, 16(2), 137-150.

Prado, R., y del Águila, M. (2003). Diferencia en la resiliencia según género y nivel socioeconómico en adolescentes. Persona, 6, 179-196.

Ramis, Y., Torregrosa, M., Viladrich, M. C. y Cruz, J. (2010). Adaptación y validación de la versión española de la Escala de Ansiedad Competitiva SAS-2 para deportistas de iniciación. Psicothema, 22(4), 1004-1009. Retrieved from http://dialnet.unirioja.es/servlet/articulo?codigo=3320325yinfo=resumenyidioma=SPA

Ramis, Y., Torregrosa, M., y Cruz, J. (2013). Revisitando a Simon y Martens: la ansiedad competitiva en deportes de iniciación. Revista de Psicología Del Deporte, 22(1), 77-83.

Rodríguez, J. M., y Granero-Gallegos, A. (2004). Estado de ánimo. autoconfianza y ansiedad precompetitiva en tiro deportivo. Espiral. Cuadernos del profesorado, 711), 13-23.

Rosenberg, A. R., Syrjala, K. L., Martin. P. J., Flowers. M. E., Carpenter. P. A., Salit. R. B., Baker, K. S., y Lee, S. J. (2015). Resilience. Health and quality of life among long term survivors of hematopoietic cell transplantation. Cancer, 121(23), 4250-4257.

Souri, H., y Hasanirad, T. (2011). Relationship between resilience, optimism and psychological well-being in students of medicine. Procedia-Social and Behavioral Sciences, 30, 1541-1544. https://doi.org/10.1016/j. sbspro.2011.10.299

Sousa, J. (2006). As famílias como projectos de vida: 0 desenvolvimento de competências resilientes na conjugalidade e na parentalidade. Saber e Educar. 11, 41-47

Sun, J., y Stewart, D. (2007). Age and Gender Effects on Resilience in Children and adolescents. International Journal of Mental Health Promotion, 944), 16-25. https://doi.org/10.1080/14623730.2007.9721845

Tabernero, B., y Márquez, S. (1993). Diferencias de sexo y edad en los componentes de la ansiedad competitiva. Apunts: educación física y deportes, 34, 68-72.

Thatcher, J., Thatcher, R., y Dorling, D. (2004). Gender differences in the pre-competition temporal patterning of anxiety and hormonal responses. Journal of sports medicine and physical fitness, 44(3), 300.

Vanistendael, S. (1994). La resiliencia: un concepto largo tiempo ignorado. La Infancia en el mundo. VIDE: Montevideo. 
